\title{
A local Rayleigh model with spatial scale selection for ultrasound image segmentation
}

\author{
Djamal Boukerroui \\ http://www.hds.utc.fr/ dboukerr
}

\author{
Université de Technologie de \\ Compiègne \\ Heudiasyc UMR CNRS 7253 \\ BP 20529 - 60205 Compiègne Cedex, \\ France.
}

\begin{abstract}
Ultrasound images are very noisy, with poor contrast and the attenuation of the acoustic wave in the depth of the observed medium leads to strong inhomogeneities in the image. Segmentation methods using global image statistics give unsatisfactory results. The use of local image statistics can solve effectively the problem of attenuation. The contribution of this paper is two folds. First, we propose the study of the adaptation of the global model proposed by Sarti et al. [E]]. We kept the variational framework and the Rayleigh model of the observed image statistics. Second, we propose an interesting and generic adaptive scale selection algorithm based on the Intersection of Confidence Interval rule. The latter is also applied to the local Gaussian segmentation model of Brox and Cremers. Results on realistic simulations of ultrasound images show the robustness and the superiority of the local Rayleigh model. The efficiency and the genericity of the proposed scale selection strategy is also demonstrated.
\end{abstract}

\section{Introduction}

In medical imaging, the ultrasonic wave is modelled as a progressive plane mechanical wave. When this wave encounters an interface between two tissues with different acoustic characteristics, a part of the incident wave is reflected (specular echoes) in the direction of the probe. Along with these specular echoes, backscattered echoes are added by the microscopic structure of the medium. Backscattering is the origin of the speckle phenomenon, which characterizes ultrasound imaging with a granular appearance. The speckle is a multiplicative noise, strongly correlated and more importantly, with non Gaussian statistics. These characteristics differ greatly from the traditional assumption of white additive Gaussian noise, often taken in ultrasound image segmentation, which leads to reduction of the effectiveness of the methods. Thus, several researchers have studied the statistics of the envelope image of the received echo signal in order to obtain processing algorithms specific to ultrasonic data $[\square, \square]$. Several distribution families have been proposed in the literature: specific models

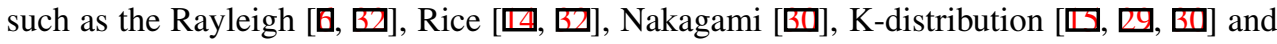
more general models, the Homodyne K-distribution [ $\mathrm{\theta}]$ and the more recently RIIG [四, $\square$ ]. Notice the large variability of the proposed models, which is due to the strong dependence of the observed statistics on the density of scatters and on their spatial distribution (uniform 
or random) in the analysed tissue [B]]. All these models are for the envelope of the received echo signal (i.e before interpolation, log-compression and Time-Gain-Compensation). Thus, the validity of such models on ultrasound images acquired under clinical conditions is ques-

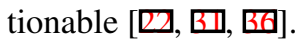

Image segmentation in general is an ill-posed problem in the sense of Hadamard and difficult to solve. This problem is especially difficult when the data to be processed are medical in nature. The literature on the subject is abundant and the performances of the proposed techniques are highly dependent on the quality of data [ $\mathbb{Z}$, B]]. Ultrasound data are very noisy, with poor contrast, and often presents missing boundaries of the object of interest due to problems of specular reflection, shadows, signal dropout and attenuation. As a consequence, conventional intensity gradient-based methods have had limited success on typical clinical images [ $\mathbb{\Omega}]$. Solutions using the phase information, theoretically invariant to image contrast, are successfully used in $[\square, \sigma]$. Note also that segmentation methods based on global statistical models, regardless of the used framework, fail on this type of data, mainly because of the attenuation problem. Adaptive solutions robust to attenuation exist in the literature $[\mathrm{d}, \boldsymbol{Q}, \boldsymbol{\nabla}, \mathbb{\Xi}]$. Local image statistics were used for the estimation of the segmentation model's parameters.

Recently, there has been a reinvestigation of the use of local statistics by the image segmentation community, but in a variational framework [Q, 四, 四, 四]. For instance, the adaptation of the binary model of Chan and Vese $[\boldsymbol{Q}]$ is presented in $[\mathbb{}[]$. A comprehensive formalization of generic local segmentation models is proposed in [ष]], with examples of local energies derived from global ones. Also, a statistical interpretation of the piecewise smooth segmentation model of Mumford-Shah using local Gaussian models has been proposed in [Q]. An interesting alternative with local Gaussian distributions is also proposed in [B]]. These recent studies show a better behavior of these local models on images with strong intensity inhomogeneities. This contribution falls under this context. We propose the study of adaptation of the model proposed by Sarti et al. [ $\mathbb{Z}$ ]. The latter assumes a global Rayleigh model envelope image statistics. The reader is also referred to [ $[\mathbf{D}]$ for a generalisation to the exponential family.

\section{Segmentation model}

Let $I: \Omega \rightarrow \mathbb{R}^{+}$denote a given observed image and $\mathcal{C}$ be a closed contour represented as the zero level set of a signed distance function $\phi$, i.e., $\mathcal{C}=\{\mathbf{x} \mid \phi(\mathbf{x})=0, \mathbf{x} \in \Omega\}$. The interior $\Omega_{i}$ and the exterior $\Omega_{e}$ of $\mathcal{C}$ are defined by a smooth approximation of the Heaviside function respectively by: $H_{i}(\phi)=H(\phi)$ and $H_{o}(\phi)=1-H(\phi)$. Image intensities are supposed to be independent realisation of random variables with a certain probability density function (pdf) $p(I)$. We seek the partition of $\Omega$ that maximizes the likelihood function of the observed data. Given the independence assumption, this leads to the minimization of the following energy function $[\mathbb{Q}]$

$$
E(\phi)=-\sum_{r \in\{i, o\}} \int_{\Omega} H_{r}(\phi) \log p(I(\mathbf{x})) d \mathbf{x}+\lambda \int_{\Omega} \delta(\phi)|\nabla \phi| d \mathbf{x}
$$

where the first two terms are the data terms and the last one is a length regularisation term, added in order to keep the curve smooth, with a positive weight penalty $\lambda$. We will further 
assume that the random intensity $I(\mathbf{x})$ follows a Rayleigh pdf with a parameter $\sigma^{2}$ :

$$
p(I(\mathbf{x}))=\frac{I(\mathbf{x})}{\sigma^{2}} \exp \left(-\frac{I(\mathbf{x})^{2}}{2 \sigma^{2}}\right) \text { and } \widehat{\sigma^{2}} \widehat{M L}=\frac{\int_{\Omega_{r}} I(\mathbf{x})^{2} d \mathbf{x}}{2 \int_{\Omega_{r}} d \mathbf{x}},
$$

where $\widehat{\sigma^{2}}$ ML is a Maximum Likelihood estimates under the assumption that all the observed pixels in the domain $\Omega_{r}$ are identically distributed. In the work of Sarti et al. [ $[\mathbb{d}$ ], only two global domains were used, $\Omega_{i}$ for the inside and $\Omega_{e}$ for the outside pixels. Therefore the hypothesis of identically distributed observations is generally false for ultrasound images because of the presence of strong intensity inhomogeneities due to attenuation and other factors. However, the assumption remains true if the estimate is made locally in a region centered around each pixel of the domain $\Omega$. Thus the energy corresponding to the inside term of (1) is given by:

$$
\begin{aligned}
E_{i}(\phi) & =\int_{\Omega} H(\phi)\left[\frac{I(\mathbf{x})^{2}}{2 \sigma_{i}^{2}(\mathbf{x})}+\log \left(\sigma_{i}^{2}(\mathbf{x})\right)\right] d \mathbf{x} \\
\text { and } \sigma_{i}^{2}(\mathbf{x}) & =\frac{\int_{\Omega} H(\phi) K(\mathbf{x}-\xi) I(\xi)^{2} d \xi}{2 \int_{\Omega} H(\phi) K(\mathbf{x}-\xi) d \xi},
\end{aligned}
$$

here $K(\cdot)$ is any given kernel defining the spatial locality around the position $\mathbf{x}$. In this work a Gaussian kernel with a standard deviation $\sigma_{K}$ is used. For computing the Euler-Lagrange equation of (3), we employ the Gâteaux derivative. Following [ब], for any point $\mathbf{x}$ and for any perturbation $\psi(\mathbf{x})$ we have:

$$
\begin{aligned}
\left.\frac{\partial E_{i}(\phi+\varepsilon \psi)}{\partial \varepsilon}\right|_{\varepsilon \rightarrow 0} & =\int_{\Omega} \delta(\phi(\mathbf{x}))\left[\frac{I(\mathbf{x})^{2}}{2 \sigma_{i}^{2}(\mathbf{x})}+\log \left(\sigma_{i}^{2}(\mathbf{x})\right)\right] \psi(\mathbf{x}) d \mathbf{x} \\
& +\int_{\Omega} H(\phi(\mathbf{x}))\left[\frac{2 \sigma_{i}^{2}(\mathbf{x})-I(\mathbf{x})^{2}}{2 \sigma^{4}(\mathbf{x})}\right] \sigma_{\phi}^{2}(\mathbf{x}) d \mathbf{x} \\
\text { where } \quad \sigma_{\phi}^{2}(\mathbf{x}) & =\frac{\int_{\Omega} \delta(\phi(\xi)) K(\mathbf{x}-\xi)\left(I(\xi)^{2}-\sigma_{i}^{2}(\mathbf{x})\right) \psi(\xi) d \xi}{2 \int_{\Omega} H(\phi(\mathbf{z})) K(\mathbf{x}-\mathbf{z}) d \mathbf{z}} .
\end{aligned}
$$

Using the following notations:

$$
\begin{aligned}
& F_{1}(\mathbf{x})=\int_{\Omega} K(\mathbf{x}-\mathbf{y}) H(\phi(\mathbf{y})) d \mathbf{y}=(K * H(\phi))(\mathbf{x}), \\
& F_{2}(\mathbf{x})=\left(\bar{K} * \frac{H(\phi)\left(2 \sigma^{2}-I^{2}\right)}{\sigma^{4} F_{1}}\right)(\mathbf{x}), F_{3}(\mathbf{x})=\left(\bar{K} * \frac{H(\phi)\left(2 \sigma^{2}-I^{2}\right)}{\sigma^{2} F_{1}}\right)(\mathbf{x}),
\end{aligned}
$$

where $\bar{K}$ is the mirror version of $K$ and $*$ is the convolution operation. The Euler-Lagrange equation for the minimization of the energy $E_{i}$, given in (3), obtained from (5) simplifies then as follows:

$$
0=\delta(\phi)\left[\frac{I(\mathbf{x})^{2}}{2 \sigma_{i}^{2}(\mathbf{x})}+\log \left(\sigma_{i}^{2}(\mathbf{x})\right)+\frac{1}{4}\left(I(\mathbf{x})^{2} F_{2}(\mathbf{x})-F_{3}(\mathbf{x})\right)\right],
$$

and can be implemented very efficiently using recursive gaussian filtering [ $\square]$ ]. The derivation of the Euler-Lagrange equation corresponding to the energy in $\Omega_{o}$ (second term in the sum in (1)) is obtained by analogy to the above equation. The gradient of the length term can be found in $[\boldsymbol{\nabla}, \mathbb{\square}]$. 


\section{Spatial scale selection}

Local region-based segmentation models are surely a better alternative to global ones in the presence of intensity inhomogeneities. Such models however may be more sensitive to initialisation if the chosen local spatial scale is not appropriate. A decrease of robustness to noise is also observed when small scales are used. To our knowledge, two pixel dependent scale selection methods have been introduced recently. Piovano and Papadopoulo defined the local scale as "the smallest one inducing an evolution speed superior to a given threshold" [ $\square]$. Yang and Boukerroui proposed that the optimal scale is "in the sense of the meansquare error minimisation of a Local Polynomials Approximation (LPA) of the observed image conditional on the current segmentation" [ $\square]$. The former is a generic approach and the latter is based on an Intersection of Confidence Intervals (ICI) algorithm, which finds the optimal LPA estimate of the image. Since, the LPA assumes an additive gaussian noise model, the performances of this method may decrease when the noise model is not additive gaussian. In this section, we first recall Piovano and Papadopoulo's methods and then introduce a new generic ICI rule for the spatial scale selection. Both methods can estimate continuous scales but for efficiency reasons, the search is generally limited to a finite set of ordered scale values $\mathbf{h}=\left\{h_{1}<h_{2}<\ldots<h_{J}\right\}$.

\subsection{Evolution speed threshold based approach}

In [ $\square]$, the idea is to find the most salient scale to make the contour locally evolve. It is defined as the smallest one inducing an evolution speed superior to a given threshold $\varepsilon$ :

$$
h^{*}(\mathbf{x})=\inf _{h \in \mathbf{h}}\left\{h:\left|\partial_{t} \phi(\mathbf{x})\right|>\varepsilon \quad \text { and } \quad \sigma_{K}=h\right\} .
$$

Thus, at each iteration of the level set evolution, the optimal scale is found for every image pixel along the zero level set function, by increasing $\sigma_{K}(\mathbf{x})$ from the minimum $h_{1}$ to the maximum $h_{J}$, until the absolute value of the evolution speed is superior to $\varepsilon$. It is unclear how to fix the positive constant $\varepsilon$.

\subsection{ICI rule}

In the following section, we introduce a new alternative approach in order to choose a good value for the spatial scale at every pixel location along the contour. Our approach is based on the idea of choosing the largest scale that gives the best estimate of the segmentation model parameters. The approach is generic and can be applied to any parametric pdf. For the sake of clarity, we detail the development for the Rayleigh case only and we give the main results for the Gaussian case.

\subsubsection{The Rayleigh case}

It can be shown that the ML estimator of $\sigma^{2}$ given in eq. (2) is efficient. It is therefore unbiased and has the lowest possible variance defined by the Cramer-Rao bound. It is also asymptotically Gaussian as it is a ML estimator: for $n \rightarrow \infty \widehat{\sigma^{2}} \rightarrow \mathcal{N}\left(\sigma^{2}, \sigma^{2} / n\right)$ where $n=\int_{\Omega_{r}} d \mathbf{x}$ in our context. Therefore we can estimates a Confidence Interval on the estimates 
of $\sigma^{2}$ by :

$$
P\left(\sigma^{2} \in\left[\frac{\widehat{\sigma^{2}}}{1+\frac{u_{1-\alpha / 2}}{\sqrt{n}}}, \frac{\widehat{\sigma^{2}}}{1-\frac{u_{1-\alpha / 2}}{\sqrt{n}}}\right]\right) \simeq 1-\alpha .
$$

where $u_{\alpha}$ is the $\alpha^{\text {th }}$ percentile of the standard Gaussian distribution $\mathcal{N}(0,1)$. Equivalently, the confidence interval for the the estimator $\sigma_{i}^{2}(\mathbf{x})$ given in eq. (4) is given by eq. (8) with

$$
\widehat{\sigma^{2}}=\sigma_{i}^{2}(\mathbf{x}) \quad \text { and } \quad n=\frac{\left(\int_{\Omega} H(\phi) K(\mathbf{x}-\xi) d \xi\right)^{2}}{\int_{\Omega}(H(\phi) K(\mathbf{x}-\xi))^{2} d \xi} .
$$

Therefore in an ideal situation, where the observed data is identically distributed, the bigger $n$ is, the better is the estimates of $\sigma^{2}$. Bear in mind that the hypothesis of identically distributed data in the local window will become less and less valid as the scale of the kernel $K$ grows and will lead to an increasingly biased estimations. This means that there exists a bias-variance balance that gives the ideal scale. We can make use of the ICI algorithm to search for the largest local window (minimising variance) that gives us the best estimate of $\sigma^{2}$ (minimising bias). First, the estimates $\widehat{\sigma^{2}} h$ and their corresponding CIs are calculated for all $h \in \mathbf{h}$. Then, the ICI rule identifies the best scale $h^{*}$ as the largest in $\mathbf{h}$ that has a non empty intersection of CIs with all scales in $\left\{h_{i} \in \mathbf{h}, h_{i}<h^{*}\right\}$. The ICI algorithm is defined by the following steps $[\square, \square]$ :

1. Define a sequence of confidence intervals $Q_{i}=Q\left(h_{i}\right)$ with their lower $L_{i}$ and upper bounds $U_{i}$ using eq. 8 .

2. For $i=1,2, \ldots, J-1$, let

$$
\begin{aligned}
\bar{L}_{i+1} & =\max \left\{\bar{L}_{i}, L_{i+1}\right\}, & \bar{L}_{1} & =L_{1}, \\
\underline{U}_{i+1} & =\min \left\{\underline{U}_{i}, U_{i+1}\right\}, & \bar{U}_{1} & =U_{1} .
\end{aligned}
$$

According to these formulas, $\bar{L}_{i+1}$ and $\underline{U}_{i+1}$ are respectively non-decreasing and nonincreasing sequences.

3. The ICI rule is finding the largest $i$, when $\bar{L}_{i} \leq \underline{U}_{i}, \quad i=1,2, \ldots, J$, is still satisfied.

\subsubsection{The Gaussian case}

When the observed image intensities $I(\mathbf{x})$ are supposed to follow local Gaussian distributions [日], we can base our scale estimation on the confidence intervals of the local means. Recall that if $X \sim \mathcal{N}\left(\mu, \sigma^{2}\right)$ and given an independent and identically distributed sample of size $n$ then:

$$
P\left(\mu \in\left[\bar{X}-\frac{S}{\sqrt{n}} t_{n-1,1-\alpha / 2} \quad, \quad \bar{X}+\frac{S}{\sqrt{n}} t_{n-1,1-\alpha / 2}\right]\right)=1-\alpha .
$$

where $S^{2}$ is the unbiased estimate of the variance and $t_{n, \alpha}$ is the $\alpha^{\text {th }}$ percentile of a Student's t-distribution of $n$ degrees of freedom [四]. Thus, Eq. 9 gives us a set of CIs that can be utilised in an ICI algorithm in order to select the best scale for the estimation of the local means. Note that eq. 9 is exact $\forall n$. By use of the Central Limit Theorem, eq. 9 can be used as an approximation of the CIs by replacing Student's percentiles by the Gaussian one in the general non gaussian case. 


\subsubsection{The scale selection procedure}

The above proposed ICI rule is used to estimate the best spatial kernel at every pixel location along the contour. Note however that the ICI rule will produce two scale values, $h_{i}^{*}$ and $h_{o}^{*}$ corresponding for the inside region $\Omega_{i}$ and the outside region $\Omega_{o}$ respectively. In [ $\left.\square\right]$ ] the used scale corresponds to the maximum between the two values. In this work we followed the same procedure as in $[\mathrm{G}]$ until converges, we ran few iterations by replacing the maximum by an average filtering and then by using directly the estimated scales: $h_{i}^{*}$ for the inside energy term and $h_{o}^{*}$ for the outside energy term.

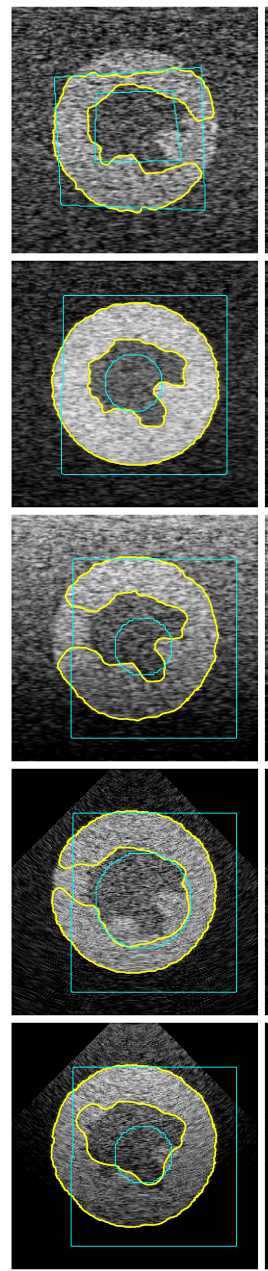

$\sigma_{K}=20$
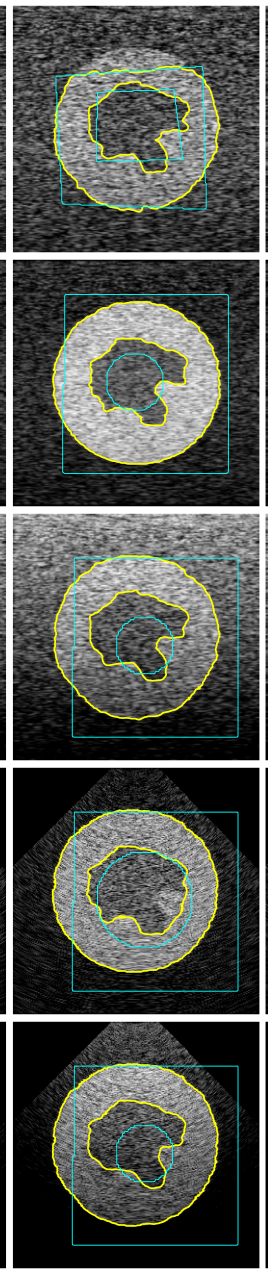

$\sigma_{K}=40$
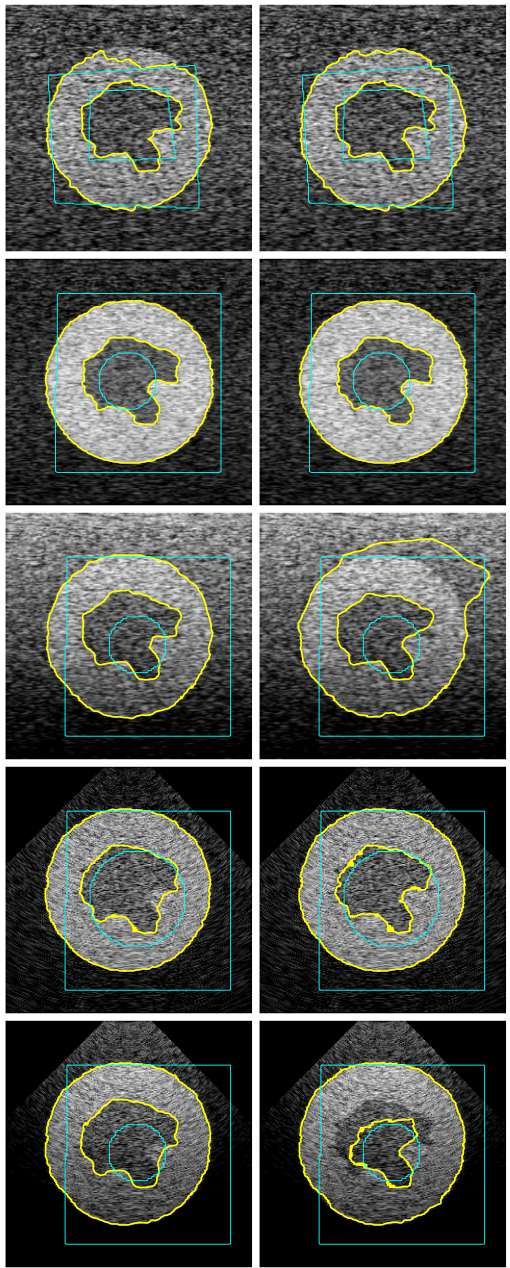

$\sigma_{K}=55$
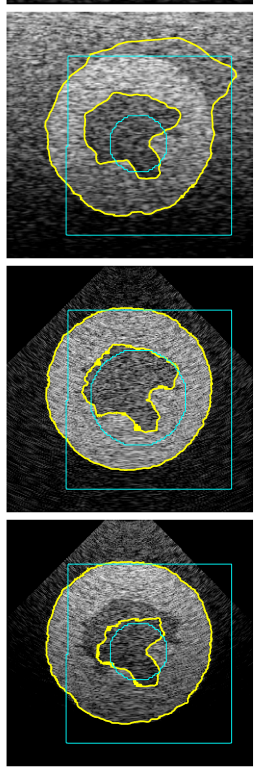

$\sigma_{K}=70$
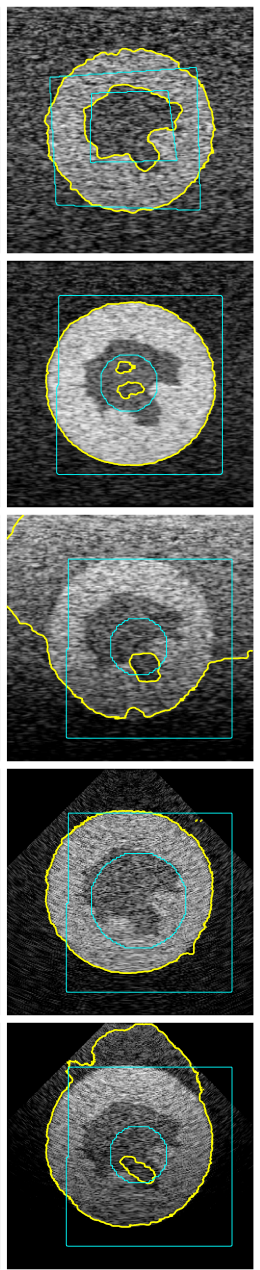

$\sigma_{K}=200$

Figure 1: Illustrative segmentation results of the local Rayleigh model on simulated US images with different: tissues characteristics, attenuation level and log compression parameters. Thin lines show the (4 different) initialisations. For better contrast, the images are displayed after the logarithmic compression of the envelope but the image segmentation algorithm uses the data before compression. 

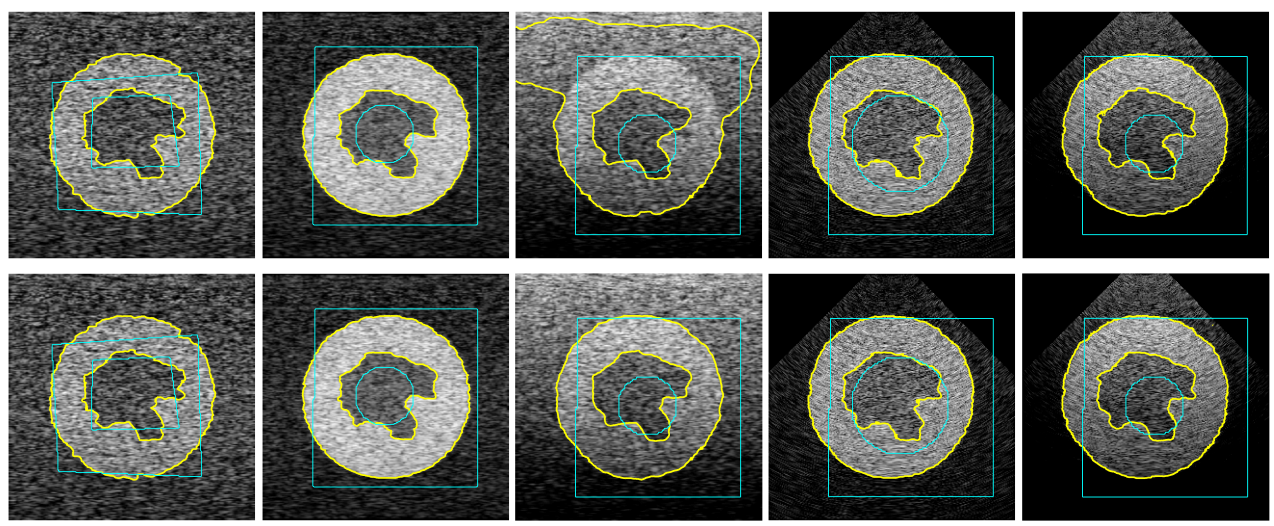

Figure 2: Illustrative segmentation results of the proposed local Rayleigh model with spatial scale selection. First line: scale selection method of $[\square]$ with $\varepsilon=0.5$. Second line: the proposed ICI based approach with $\alpha=0.05$. These results are to be compared to the one shown in Fig.1. The used set $\mathbf{h}=\{5,10,15,20,25,30,35,40,45,50,60,70,80\}$ for an image size of $256 \times 256$.

\section{Results}

In order to demonstrate the usefulness of the proposed approach and quantify its performances, we chose to test it on realistic US simulations. To this end, we have used the simulation program Field-II [ $[\square, \square]$, to synthesize phantom data with known ground truth. A linear scan of a first phantom (PH1) was done with a 290 elements transducer using 64 active elements. The scatterers in the phantom were randomly distributed within the phantom of $80 \times 80 \times 15 \mathrm{~mm}$ cube size. 128 lines were simulated at $5 \mathrm{Mhz}$. The second phantom (PH2) of size $100 \times 100 \times 15 \mathrm{~mm}$ cube was placed at $10 \mathrm{~mm}$ depth from the transducer surface, and was scanned with a $7 \mathrm{MHz} 128$ elements phased array transducer. The images consist of 128 lines with 0.7 degrees between lines. Hanning apodization in transmit and receive was used in all experiments. Two scatterers amplitudes with three levels of tissue attenuations were simulated for both phantoms. We also used several $\mathrm{dB}$ ranges for the envelope logarithmic compression to simulate different image contrasts. Some typical images are shown in Fig. 1.

The first experiment demonstrates the behaviour of the proposed local Rayleigh approach when different sizes of the local spatial kernel and different initialisations were used (see. Fig. 1). Small local scales generally decrease the capture range of the active contour and also increase the number of local minima. Big scale values lead to unsatisfactory results in the presence of intensity inhomogeneities. Figure 2 shows the corresponding results to the one shown in Fig. 1, when an automatic scale selection procedure is used. Observe that the proposed ICI rule based approach outperform the method in [ $\square]$ ].

In order to understand better the behaviour of the two automatic scale selection algorithms, Fig. 3 shows the contour evolution and the estimated scale maps for the two approaches on the image of Fig. 2(middle column). Although, the interpretation of the scale maps estimated by the proposed ICI rule is possible, it is difficult to understand the ones obtained with Piovano and Papadopoulo's method. Indeed, our approach is driven by a biasvariance tradeoff for the local estimation of the segmentation model parameter, conditional on the current segmentation map. This is why for example we get higher scales when the algorithm converges (except the last iteration where we deliberately reduce the scales in order 
to increase accuracy, see sec.3.2.3). Furthermore, it is easier to set the value of $\alpha$, because it is more interpretable, then the threshold $\varepsilon$. For this example, the segmentation fail, with Piovano and Papadopoulo's scale selection method, because the estimated scales are too big in the upper part of the image.

The quantitative evaluation of the proposed approach compared to ground truth are summarized in Table 1. It shows statistics of the Dice similarity Coefficient DSC $\left(S, S_{\text {ideal }}\right)=$ $2 \frac{\left|S \cap S_{\text {ideal }}\right|}{|S|+\left|S_{\text {ideal }}\right|}$ and the Mean Absolute Distance (MAD) [0] of segmentation results of the 60 simulated images of the two phantoms with 4 different initialisations $(60 \times 4=240$ results $)$. The closer the DSC and the MAD values to 1 and 0 respectively, the better is the segmentation. The table shows the minimum, the three Quartiles, the maximum and the interquartile range (IQR) of the measurements, for six sizes of the spatial kernel $K(\cdot)$. This table clearly shows that local image statistics should be used in the presence of intensity inhomogeneities. The minimum and the $\mathrm{Q}_{1}$ values of DSC and the maximum and the $\mathrm{Q}_{3}$ values of MAD (indicating the worst cases) are very informative. Notice also the IQR values, a robust measure of dispersion. The worst results are for very large values of $\sigma_{K}$. In this case the algorithm's behaviour is similar to the global method of Sarti et al. [ $[\mathbb{Q}]$. Notice that both scale selection methods performed pretty well. These measurements indicates a superiority of the new proposed scale section methodology on Piovano and Papadopoulo's approach. The interpretation of these data suggests the use of local statistics for the segmentation of ultrasound images.
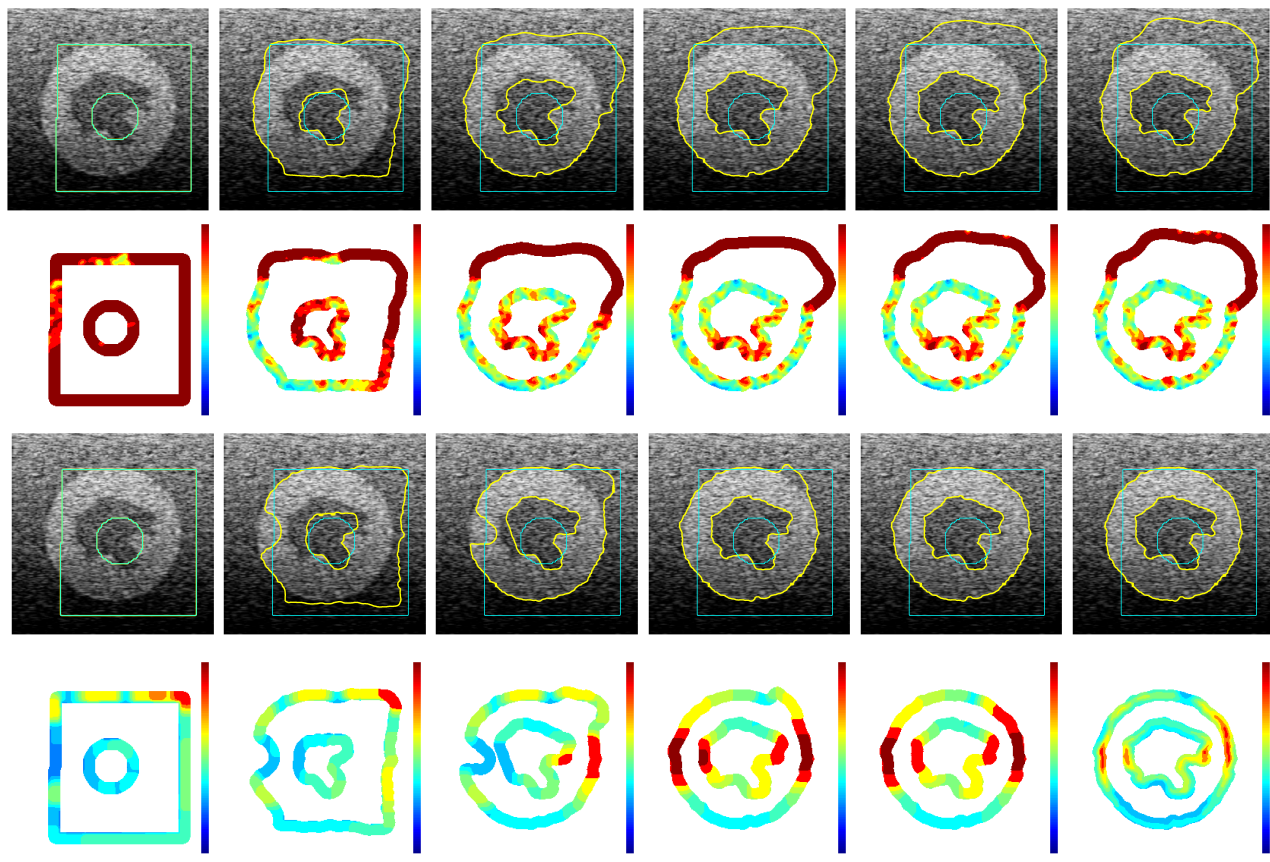

Figure 3: Behaviour of the two automatic scale selection algorithms. $1^{\text {st }}$ and $3^{\text {rd }}$ lines: contour evolution of the local Rayleigh segmentation model for iterations 1, 10, 25, 50, 75 and 120 , respectively when using the scales shown in the $2^{\text {nd }}$ (estimated with Piovano and Papadopoulo's method. see sec.3.1) and the $4^{\text {th }}$ (obtained with the proposed ICI rule) lines. 


\begin{tabular}{|c||cccccc|cccccc|}
\hline \multicolumn{1}{|c||}{} & \multicolumn{5}{c|}{$\mathrm{DSC} \times 100$} & \multicolumn{5}{c|}{ MAD (in pixels) } \\
\hline & min & $\mathrm{Q}_{1}$ & $\mathrm{Q}_{2}$ & $\mathrm{Q}_{3}$ & $\max$ & $\mathrm{IQR}$ & $\min$ & $\mathrm{Q}_{1}$ & $\mathrm{Q}_{2}$ & $\mathrm{Q}_{3}$ & max & IQR \\
\hline$\sigma_{K}=20$ & 56.4 & 83.5 & 89.2 & 93.5 & $\mathbf{9 6 . 5}$ & 10.0 & 1.10 & 1.33 & 2.09 & 3.87 & 20.51 & 2.54 \\
$\sigma_{K}=30$ & 60.2 & 90.3 & 95.8 & 96.0 & $\mathbf{9 6 . 5}$ & 5.8 & 1.11 & 1.29 & $\mathbf{1 . 3 8}$ & 1.95 & 23.70 & 0.66 \\
$\sigma_{K}=40$ & 46.3 & $\mathbf{9 5 . 4}$ & $\mathbf{9 5 . 9}$ & $\mathbf{9 6 . 1}$ & $\mathbf{9 6 . 5}$ & 0.7 & 1.11 & $\mathbf{1 . 2 5}$ & $\mathbf{1 . 3 8}$ & $\mathbf{1 . 4 8}$ & $\mathbf{2 3 . 3 9}$ & $\mathbf{0 . 2 3}$ \\
$\sigma_{K}=55$ & 54.2 & $\mathbf{9 5 . 4}$ & 95.7 & 96.0 & $\mathbf{9 6 . 5}$ & $\mathbf{0 . 6}$ & 1.10 & 1.29 & 1.39 & 1.53 & 24.76 & $\mathbf{0 . 2 3}$ \\
$\sigma_{K}=70$ & $\mathbf{6 2 . 7}$ & 93.7 & 95.1 & 95.7 & 96.4 & 2.0 & $\mathbf{1 . 0 6}$ & 1.37 & 1.53 & 1.74 & 24.19 & 0.37 \\
\hline$\sigma_{K}=200$ & 46.1 & 54.5 & 79.3 & 85.6 & 95.1 & 31.1 & 1.29 & 2.26 & 4.06 & 19.76 & 65.39 & 17.50 \\
\hline \hline$\sigma_{K}=h^{*}$ & 62.4 & 93.6 & 95.9 & $\mathbf{9 6 . 2}$ & $\mathbf{9 6 . 5}$ & 2.6 & 1.11 & $\mathbf{1 . 2 1}$ & $\mathbf{1 . 3 1}$ & 1.90 & 24.27 & 0.69 \\
$\sigma_{K}=h_{\text {ICI }}^{*}$ & $\mathbf{6 8 . 2}$ & $\mathbf{9 5 . 5}$ & $\mathbf{9 6 . 1}$ & $\mathbf{9 6 . 2}$ & $\mathbf{9 6 . 5}$ & $\mathbf{0 . 7}$ & $\mathbf{1 . 0 9}$ & 1.29 & 1.36 & $\mathbf{1 . 4 7}$ & $\mathbf{2 1 . 5 4}$ & $\mathbf{0 . 1 7}$ \\
\hline
\end{tabular}

Table 1: Statistics of the DSC and the MAD measures obtained on 60 images with 4 different initialisations. $\sigma_{K}$ is the standard deviation of the spatial gaussian kernel. $h^{*}$ is the proposed segmentation approach with the scale selection in $[\square] . h_{I C I}^{*}$ is the proposed approach with the proposed ICI scale selection procedure. $\sigma_{K}=200$ is equivalent to the method in Sarti et al. [지].

\begin{tabular}{|c||cccccc|cccccc|}
\hline \multicolumn{1}{|c||}{} & \multicolumn{6}{c|}{$\mathrm{DSC} \times 100$} & \multicolumn{5}{c|}{ MAD (in pixels) } \\
\hline & $\min$ & $\mathrm{Q}_{1}$ & $\mathrm{Q}_{2}$ & $\mathrm{Q}_{3}$ & $\max$ & $\mathrm{IQR}$ & $\min$ & $\mathrm{Q}_{1}$ & $\mathrm{Q}_{2}$ & $\mathrm{Q}_{3}$ & $\max$ & $\mathrm{IQR}$ \\
\hline$\sigma_{K}=10$ & 62.5 & 72.0 & 90.5 & 94.6 & 96.1 & 22.7 & $\mathbf{1 . 1 1}$ & 3.86 & 15.06 & 17.93 & 34.50 & 14.07 \\
$\sigma_{K}=20$ & $\mathbf{6 5 . 9}$ & 88.4 & 94.8 & 95.8 & 96.3 & 7.5 & 1.14 & 1.61 & 7.78 & 19.80 & 30.33 & 18.19 \\
$\sigma_{K}=30$ & 49.4 & $\mathbf{9 3 . 9}$ & $\mathbf{9 5 . 6}$ & $\mathbf{9 5 . 9}$ & $\mathbf{9 6 . 5}$ & $\mathbf{1 . 9}$ & 1.12 & $\mathbf{1 . 4 3}$ & $\mathbf{1 . 9 4}$ & 12.61 & 27.49 & 11.18 \\
$\sigma_{K}=40$ & 58.8 & 89.8 & 94.8 & 95.7 & 96.4 & 5.9 & 1.15 & 1.48 & 2.02 & 10.78 & $\mathbf{2 5 . 2 0}$ & 9.31 \\
$\sigma_{K}=55$ & 54.0 & 80.9 & 93.3 & 95.4 & 96.0 & 14.5 & 1.26 & 1.51 & 2.02 & $\mathbf{1 0 . 0 8}$ & 27.43 & $\mathbf{8 . 5 6}$ \\
$\sigma_{K}=70$ & 50.3 & 72.3 & 91.7 & 95.1 & 95.8 & 22.8 & 1.26 & 1.64 & 2.39 & 15.10 & 51.42 & 13.46 \\
$\sigma_{K}=200$ & 42.0 & 50.6 & 64.5 & 80.9 & 95.0 & 30.3 & 1.51 & 10.43 & 20.26 & 41.01 & 72.96 & 30.58 \\
\hline \hline$\sigma_{K}=h^{*}$ & 65.6 & 79.5 & 95.7 & $\mathbf{9 6 . 0}$ & $\mathbf{9 6 . 6}$ & 16.5 & $\mathbf{0 . 9 9}$ & 1.44 & 12.44 & 22.32 & 34.59 & 20.87 \\
$\sigma_{K}=h_{\mathrm{ICI}}^{*}$ & $\mathbf{6 6 . 1}$ & $\mathbf{9 4 . 4}$ & $\mathbf{9 5 . 8}$ & $\mathbf{9 6 . 0}$ & 96.5 & $\mathbf{1 . 6}$ & 1.14 & $\mathbf{1 . 3 4}$ & $\mathbf{1 . 5 5}$ & $\mathbf{1 1 . 6 7}$ & $\mathbf{2 7 . 5 7}$ & $\mathbf{1 0 . 3 3}$ \\
\hline
\end{tabular}

Table 2: Results on the same data set with the local Gaussian approach proposed in [曰]. Note that the last two lines make use of the scale section methods detailed in sec. 3 .

For the sake of completeness and comparison, we present the performance of the approach presented in [ $\mathrm{Q}]$, which also use local image statistics modelled, however, by local Gaussian pdfs. Figure 4 and Table 2 show results for the local Gaussian case respectively equivalent to the one in Fig. 2 and Table 1 for the local Rayleigh. Although the DSC measure is not very discriminative between the two models, the MAD shows clearly that the Gaussian model is less competitive in comparison to the local Rayleigh one. This observation is also demonstrated by Fig. 4. Here too, the used of an automatic scale selection strategy improves significantly the segmentation results over the single scale segmentation algorithm. Finally, it is important to mention that in the Gaussian case there was a need to increase the contribution of the regularisation of the length term (in comparison to the Rayleigh case) in order to obtain acceptable results.

\section{Conclusion}

This paper presents the adaptation of the global model proposed by Sarti et al. [ $\mathbb{Q}]$ ], in order to use local image statistics. We kept the variational framework and the Rayleigh pdf to model the observe intensities of the ultrasound envelope image. We have also proposed an interesting adaptive scale selection procedure based on the ICI rule. Results on realistic simulations of ultrasound images show the robustness and the superiority of the proposed segmentation approach in comparison to $[\boldsymbol{\square}, \mathbb{Z}]$. The efficiency and the genericity of the proposed scale selection strategy is also demonstrated. 

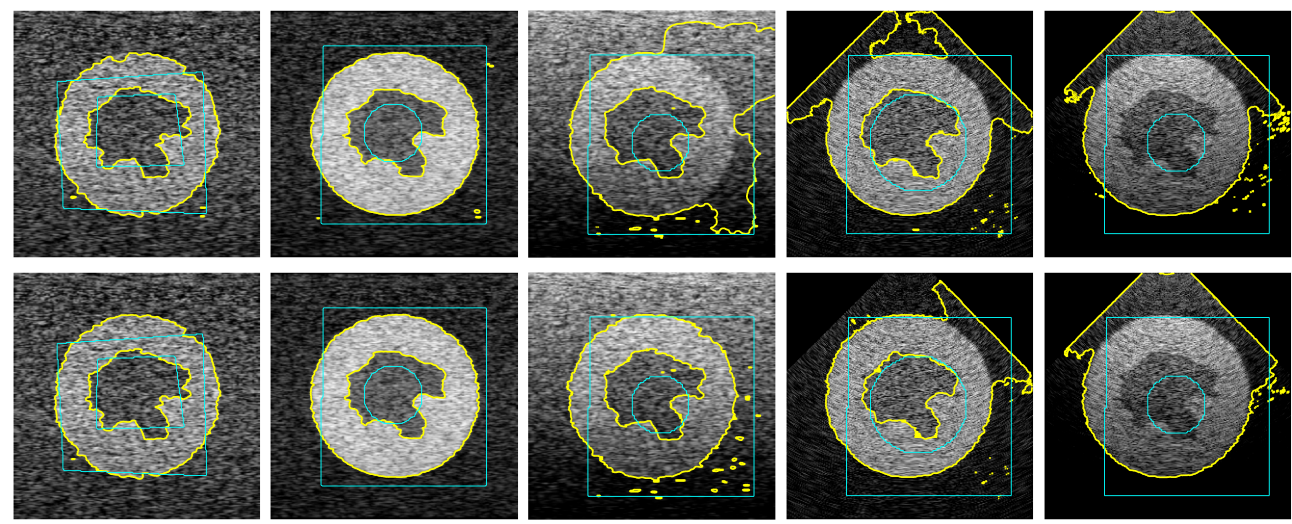

Figure 4: Same results as in Fig.2 but for the local Gaussian model. First line: scale selection of $[\square]$ with $\varepsilon=0.5$. Second line: with the proposed ICI based approach with $\alpha=0.01$.

\section{References}

[1] E. A. Ashton and K. J. Parker. Multiple resolution bayesian segmentation of ultrasound images. Ultrasonic Imag., 17(4):291-304, October 1995.

[2] A. Belaid, D. Boukerroui, Y. Maingourd, and J.-F. Lerallut. Phase-based level set segmentation of ultrasound images. IEEE Trans. Inf. Tech. Biomed., 15(1):138-147, 2011.

[3] D. Boukerroui, O. Basset, A. Baskurt, and J.A. Noble. Segmentation of echocardiographic data. Multiresolution 2D and 3D algorithm based on gray level statistics. In MICCAI, pages 516-524, Cambridge, England, 1999. Springer-Verlag.

[4] D. Boukerroui, A. Baskurt, J.A. Noble, and O. Basset. Segmentation of ultrasound images-multiresolution 2D and 3D algorithm based on global and local statistics. Pattern Recognit. Lett., 24:779-790, 2003.

[5] T. Brox and D. Cremers. On local region models and a statistical interpretation of the piecewise smooth Mumford-Shah functional. Int. J. Comput. Vis., 84(2):184-193, 2009.

[6] C. B. Burckhardt. Speckle in ultrasound B-mode scans. IEEE Trans. Sonics and Ultra., SU-25(1):1-6, 1978.

[7] V. Chalana, D. T. Linker, D. R. Haynor, and Y. Kim. A multiple active contour model for cardiac boundary detection on echocardiographic sequences. IEEE Trans. Med. Imag., 15(3):290-298, June 1996.

[8] T. F. Chan and L. A. Vese. Active contours without edges. IEEE Trans. Image Process., 10(2):266-277, 2001.

[9] V. Dutt and J. F. Greenleaf. Ultrasound echo envelope analysis using a homodyned K-distribution signal model. Ultrasonic Imag., 16(4):265-287, October 1994. 
[10] T. Eltoft. The rician inverse gaussian distribution: A new model for non-rayleigh signal amplitude statistics. IEEE Trans. Image Process., 14(11):1722-1735, November 2005.

[11] T. Eltoft. Modeling the amplitude statistics of ultrasonic images. IEEE Trans. Med. Imag., 25(2):1722-1735, February 2006.

[12] J. Geusebroek, Arnold Smeulders, and Joost van de Weijer. Fast anisotropic gauss filtering. IEEE Trans. Image Process., 12(8):938-943, 2003.

[13] A. Goldenshluger and A. Nemirovski. On spatial adaptive estimation of nonparametric regression. Math. Meth. Statistics, 6:135-170, 1997.

[14] M. F. Insana, R. F. Wagner, B. S. Garra, D. G. Brown, and T. H. Shawker. Analysis of ultrasound image texture via generalized Rician statistics. Optical Engineering, 25(6): 743-748, 1986.

[15] E. Jakeman. K-distributed noise. J. Opt. A: Pure Appl. Opt., 1:784-789, 1999.

[16] J. A. Jensen. Field: A program for simulating ultrasound systems. In 10th NordicBaltic Conf. on Biomedical Imag., volume 34, pages 351-353, 1996.

[17] J. A. Jensen and N. B. Svendsen. Calculation of pressure fields from arbitrarily shaped, apodized, and excited ultrasound transducers. IEEE Trans. Ultrason., Ferroelec., Freq. Contr., 39:262-267, 1992.

[18] Vladimir Katkovnik, Karen Egiazarian, and Jaakko Astola. Adaptive window size image de-noising based on intersection of confidence intervals (ICI) rule. J. Math. Imag. Vis., 16(3):223-235, 2002.

[19] S. Lankton and A. Tannenbaum. Localizing region-based active contours. IEEE Trans. Image Process., 17(11):2029-2039, 2008.

[20] F. Lecellier, J. Fadili, S. Jehan-Besson, G. Aubert, M. Revenu, and E. Saloux. Regionbased active contours with exponential family observations. J. Math. Imag. Vis., 36(1): 28-45, 2010.

[21] C. Li, C-Y Kao, J. C. Gore, and Z. Ding. Minimization of region-scalable fitting energy for image segmentation. IEEE Trans. Image Process., 17(10):1940-1949, 2008.

[22] N. Lin, W. Yu, and J. S. Duncan. Combinative multi-scale level set framework for echocardiographic image segmentation. Med. Image Anal., 7:529-537, 2002.

[23] M. Mulet-Parada and J. A. Noble. 2D+T acoustic boundary detection in echocardiography. Med. Image Anal., 4(1):21 - 30, 2000. ISSN 1361-8415.

[24] S. Nadarajah. Statistical distributions of potential interest in ultrasound speckle analysis. Phys. Med. Biol., 52:213-227, 2007.

[25] J. A. Noble and D. Boukerroui. Ultrasound image segmentation: A survey. IEEE Trans. Med. Imag., 25(8):987-1010, 2006.

[26] A. Papoulis and S. Pillai. Probability, Random Variables and Stochastic Processes. Mcgraw-Hill, 4th edition, 2002. 
[27] J. Piovano and T. Papadopoulo. Local statistic based region segmentation with automatic scale selection. In ECCV, pages 486-499. Springer, 2008.

[28] A. Sarti, E. Mazzini, C. Corsi, and C. Lamberti. Maximum likelihood segmentation of ultrasound images with rayleigh distribution. IEEE Trans. Ultra. Fer. Freq. Control, 52 (6):974-960, June 2005.

[29] P. M. Shankar, J. M. Reid, H. Ortega, C. W. Piccoli, and B. B. Goldberg. Use of nonRayleigh statistics for the identification of tumors in ultrasonic B-scans of the breast. IEEE Trans. Med. Imag., 12(4):687-92, 1993.

[30] P. M. Shankar, V. A. Dumane, T. George, C W Piccoli, J M Reid, F Forsberg, and B B Goldberg. Classification of breast masses in ultrasonic B scans using Nakagami and K-distributions. Phys. Med. Biol., 48(14):2229-2240, July 2003.

[31] Z. Tao and H. Tagare. Evaluation of four probability distribution models for speckle in clinical cardiac ultrasound images. IEEE Trans. Med. Imag., 25(11):1483-1491, Nov. 2006.

[32] R. F. Wagner, S. W. Smith, J. M. Sandrik, and H. Lopez. Statistics of speckle in ultrasound B-scans. IEEE Trans. Sonics and Ultra., 30(3):156-163, 1983.

[33] L. Wang, L. He, A. Mishra, and C. Li. Active contours driven by local gaussian distribution fitting energy. Singal Process., pages 2435-2447, 2009.

[34] P. N. T. Wells. Ultrasound imaging. Phys. Med. Biol., 51:R83-R98, 2006.

[35] Q. Yang and D. Boukerroui. Optimal spatial adaptation for local region-based active contours: An intersection of confidence intervals approach. In IMAGAPP, pages 8793, Algarve, Portugal, March 5-7 2011.

[36] Y. Zhu, X. Papademetris, A.J. Sinusas, and J. S. Duncan. A coupled deformable model for tracking myocardial borders from real-time echocardiography using an incompressibility constraint. Med. Image Anal., 14(3):429-448, 2010. 